\title{
Comparison of the Swelling of Treated and Untreated Hair Using an Environmental Electron Microscope (ESEM)
}

\author{
P. Hallégot ${ }^{* *}$, S. Diridollou*, V. Holloway-Barbosa**, F. Leroy** and N. J. Zaluzec ${ }^{* * *}$. \\ ${ }^{*}$ L Oréal Research, Institute for Ethnic Hair and Skin Research - Chicago, IL. - USA \\ ** L Oréal Research, Department of Physics - Aulnay - France \\ ${ }^{* * *}$ Electron Microscopy Center, Materials Science Div. Argonne National Lab - Argonne, IL - USA
}

The purpose of this work was to investigate the efficacy of ESEM for the study of hair under different relative humidity environments, as well as to measure integrity of the fiber after a relaxer treatment. In the past, the majority of work done on hair using electron microscopy has been conducted either in high vacuum on metallically coated fibers or at low voltage $(<5 \mathrm{kV})$, both conditions being used to mitigate the adverse effects of charging [1]. While providing spectacular images of the cuticle, these images do not always relate to the state of the fiber under ambient conditions and thus must be interpreted with care. Not surprisingly dehydration under high vacuum conditions is one of the most deleterious aspects of conventional SEM studies. In this work we report on measurements of the swelling of hair fiber as a function of relative humidity in an Environmental SEM.

All observations reported in this paper were carried out in a FEI Quanta 400 FEG-ESEM, operating at $25 \mathrm{kV}$, at pressures ranging from 1 to 7 Torr. Specimens were carefully attached to carbon tape and affixed to a Peltier stage whose temperature was maintained at $4{ }^{\circ} \mathrm{C}$ during these measurements. Under these conditions, the relative humidity was varied from 35 to $100 \%$. Prior to the all measurements reported, we verified both optimal imaging conditions as well as the stability of the sample as well as the absence of any visible electron beam induced damage to the surface of the cuticle. The African American hair fibers studied, were investigated in 2 states namely: virgin (untreated) and treated. The quality of the untreated hair was verified using amino acid analysis which determined that the hair was of highest quality, that is having no prior chemical treatment, either by oxidation or lanthionization. This results in a natural state which is highly curly. The same hair was also studied after treatment by straightening using a guanidine carbonate based relaxer $(\mathrm{pH}=13.5)$. The samples were treated with the relaxer for 15 minutes and then subsequently rinsed with water for $2 \mathrm{~min}$. The hair was then shampooed twice with $10 \%$ ammonium lauryl sulfate for 1 min. with another 2 minute rinse in between the shampoos. The final rinse was for 3 min. This resulted in a relaxation of its naturally curly state and a complete straightening of the hair fibers.

The study of the hair swelling with water is an established method to investigate the integrity of the fiber, hair composition and hair surface with regards to lipids [2]. In this study we measured the swelling of the fibers as a function of time under conditions from 35 through $100 \%$ relative humidity. In figures $1 \& 2$ we present images extracted from a real time video sequence of both untreated and treated hair fibers as a function of time. In both cases, measurements were carried out until such time as liquid water condensed sufficiently to obscure the fibers $(\sim 500 \mathrm{sec})$. One can observe in these figures, that the increase in diameter due to the uptake of moisture by the treated hair is significantly faster and greater than for the virgin material. Plotted in figure 3 are quantitative comparison of the measured swelling of the two fibers as a function of time at $100 \%$ relative humidity. The initial swelling rate of the untreated and treated hair was $0.02 \% / \mathrm{sec}$ and $0.08 \% / \mathrm{sec}$ 
respectively. In addition, we also note that the untreated hair reached a plateau at $\sim 4 \%$, while the treated hair continued to swell albeit at a reduced rate until such time the measurements stopped.

In conclusion, we have verified the ability of the ESEM to study hair behavior under different relative humidity environments, as well as its functionality to measure changes of the integrity of the fiber after relaxer treatment. In the future, we plan to expand this work to study the modification of the morphology and distribution of the cuticles after such humidity environments.

\section{References}

[1] J. A. Swift, Int J Cosmet Sci, 1991, 13: 143-159.

[2] A. Franbourg, et al . J Am Acad Dermatol, 2003; 48: S115-9.

[3] This work was supported in part by the U.S. DoE under BES-MS W-31-109-Eng-38 at ANL
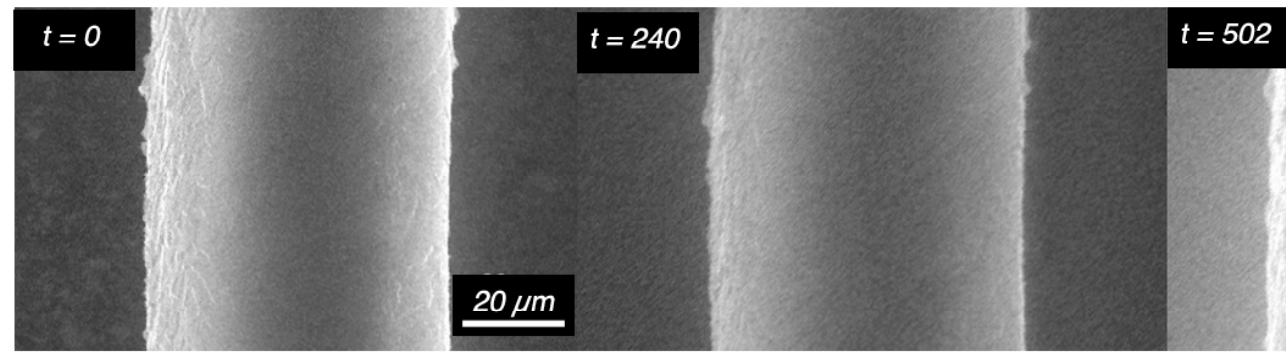

Figure 1.) Microstructural Evolution of Untreated Hair at 100\% RH vs. Time (s)

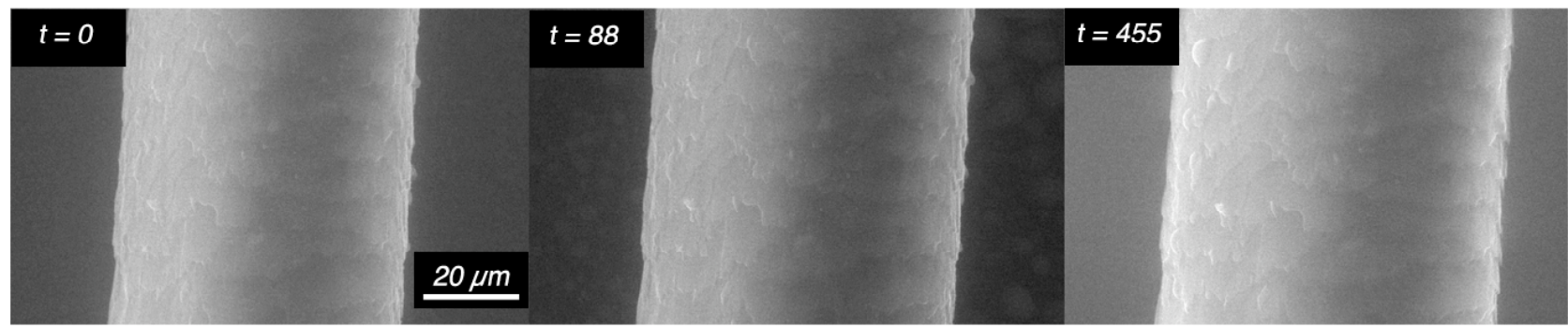

Figure 2.) Microstructural Evolution of Treated Hair at $100 \%$ RH vs Time (s) Note the change in the radial swelling relative to Figure 1.)

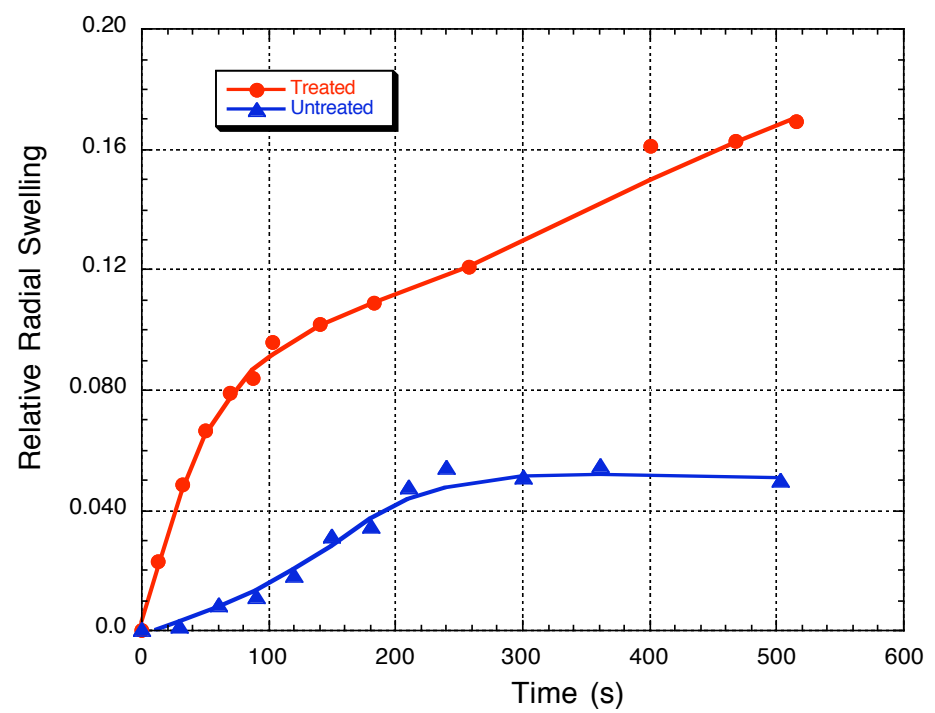

Figure 3.) Relative Radial Swelling of Hair fiber vs. Time 